\title{
Ingestão de concentrado e concentração plasmática de progesterona em vacas da raça Holandesa
}

\author{
[Concentrate intake and plasmatic progesterone concentration in Holstein cows] \\ R.M. Santos ${ }^{1,3}$, J.L.M. Vasconcelos ${ }^{2} *$ \\ ${ }^{1}$ Aluna de pós-graduação-FCAV-UNESP - Jaboticabal, SP \\ ${ }^{2}$ Faculdade de Medicina Veterinária e Zootecnia - UNESP \\ Caixa Postal 560 \\ 18618-000 - Botucatu, SP \\ ${ }^{3}$ Bolsista da FAPESP
}

\begin{abstract}
RESUMO
Vacas da raça Holandesa não-lactantes $(\mathrm{n}=7)$, que receberam $4 \mathrm{~kg} /$ dia de concentrado durante 28 dias e pasto ad libitum, foram sincronizadas com protocolo Ovsynch. No dia 0 (segunda aplicação de GnRH), as vacas foram distribuídas em dois grupos: as do grupo $2 \mathrm{~kg}$ receberam $2 \mathrm{~kg} / \mathrm{dia}$, e as do grupo $8 \mathrm{~kg}$ receberam $8 \mathrm{~kg} /$ dia de concentrado, oferecido duas vezes/dia. Os animais foram sincronizados novamente na fase 2 . Aqueles que receberam $2 \mathrm{~kg}$ de concentrado na primeira fase passaram a receber $8 \mathrm{~kg}$ na segunda fase e vice-versa. As estruturas ovarianas foram avaliadas diariamente por ultra-sonografia. Amostras de sangue para dosagem de progesterona (P4), por radioimunoensaio, foram colhidas diariamente, antes do fornecimento do concentrado e quatro horas depois, até o dia 16 do ciclo estral. Não houve diferença entre os grupos quanto ao diâmetro do folículo ovulatório no dia 0 e ao diâmetro do maior folículo no dia 16. $\mathrm{O}$ diâmetro do corpo lúteo foi maior $(\mathrm{P}<0,05)$ no dia 16 nos animais do grupo $8 \mathrm{~kg}$. Observou-se interação $(\mathrm{P}<0,05)$ da quantidade de concentrado fornecida versus dia da colheita das amostras quanto à concentração plasmática de $\mathrm{P} 4$. A concentração plasmática de $\mathrm{P} 4$, imediatamente antes do fornecimento do concentrado e quatro horas depois, foi menor no grupo 8kg, após o dia 9 do ciclo estral.
\end{abstract}

Palavras-chave: vaca de leite, progesterona, ingestão de concentrado

\begin{abstract}
Non-lactating Holstein cows ( $n=7)$ fed $4 \mathrm{~kg} /$ day of concentrate for 28 days and kept on pasture were synchronized by Ovsynch protocol. On day 0 (day of $2^{\text {nd }}$ GnRH injection), the cows were distributed in two groups: Group $2=2 \mathrm{~kg} /$ day and Group $8=8 \mathrm{~kg} /$ day of concentrate, fed twice a day. One replicate was performed (phase 1 and 2). Animals were synchronized again at the beginning of phase-2 and those that fed $2 \mathrm{~kg}$ of concentrate on phase-1, fed $8 \mathrm{~kg}$ on phase-2 and vice-versa. The ovarian structures were daily evaluated by ultrasound. Blood samples for progesterone (P4)dosage by RIA were collected daily, just before feeding the concentrate and 4 hours afterwards, until day 16 of the estrous cycle. No difference was detected between the groups in ovulatory follicle diameter on day 0 and in diameter of the largest follicle on day 16. The CL diameter was higher $(P<0.05)$ in Group 8 on day 16 of the estrous cycle. There was an interaction $(P<0.05)$ between the amount of concentrate intake and the day of blood sampling regarding P4 plasma concentration. Cows from Group 8 showed lower P4 plasma concentration, just before feed intake and four hours afterwards, after day 9 of the estrous cycle.
\end{abstract}

Keywords: dairy cow, progesterone, concentrate intake

Recebido em 9 de setembro de 2005

Aceito em 31 de agosto de 2006

*Autor para correspondência (corresponding author)

E-mail: vasconcelos@fca.unesp.br 


\section{INTRODUÇÃO}

A produção de leite por vaca tem aumentado nas últimas décadas devido à seleção genética para essa característica e ao melhor manejo nutricional oferecido aos animais. No entanto, tem-se observado declínio na eficiência reprodutiva de vacas leiteiras de alta produção (Butler, 1998; Washburn et al., 2002). Muitas podem ser as razões para essa redução da eficiência reprodutiva, incluindo diferenças no manejo reprodutivo e mudanças fisiológicas das vacas de alta produção (Lucy, 2001). Uma das características da vaca de alta produção é a maior ingestão de matéria seca (IMS), existindo uma relação $(r=0,88)$ entre a IMS e a produção de leite (Harrison et al., 1990).

Efeitos deletérios da alta IMS na eficiência reprodutiva já foram citados por vários pesquisadores (Parr et al., 1987; Dunne et al., 1999). A redução da eficiência reprodutiva em animais com alta IMS pode ser atribuída às alterações na concentração dos hormônios circulantes. Sabe-se que há uma relação inversa entre IMS e concentração plasmática de progesterona (P4) em ovelhas (Parr et al., 1993a), vacas (Vasconcelos et al., 2003) e porcas (Miller et al., 1999) e já se observou que a maior IMS aumenta o fluxo sangüíneo para a veia porta hepática (Symonds e Prime, 1989; Parr et al., 1993b; Miller et al., 1999; Sangsritavong et al., 2002). Como o fígado é o local de maior metabolização de P4 e estradiol-17ß (Parr et al., 1993a; Freetly e Ferrell, 1994), estima-se que o aumento da IMS aumente a taxa de metabolização desses hormônios esteróides, pelo aumento do fluxo sangüíneo para o fígado.

O objetivo deste experimento foi avaliar os efeitos da ingestão de diferentes quantidades de concentrado por dia sobre a concentração plasmática de progesterona nos primeiros 16 dias do ciclo estral, em vacas não-lactantes da raça Holandesa.

\section{MATERIAL E MÉTODOS}

Sete vacas da raça Holandesa, não-lactantes, submetidas a um período de adaptação de 28 dias, visando ao desenvolvimento das papilas do rúmen, foram alimentadas com $4 \mathrm{~kg}$ de concentrado por dia, à base de farelo de milho $(60 \%)$, farelo de soja $(34,5 \%)$, uréia $(1,2 \%)$ e premix mineral $(4,2 \%)$ cuja composição foi: $73 \%$ de NDT; $22 \%$ de PB e $10,5 \%$ de FDN.

Mantidos o tempo todo no pasto (MS-51,3\%; PB-4,6\%; EE-2,6\%; FB-37,4\%; NDT-54,3\%; FDN-78,6\%; FDA-43,2\%), os animais foram sincronizados, inicialmente, com o protocolo Ovsynch modificado, que consistiu na aplicação de $100 \mathrm{mcg}$ de gonadorelina ${ }^{1}$ via intramuscular $(2 \mathrm{ml})$, em dia aleatório do ciclo estral, seis dias depois da aplicação de duas doses de $25 \mathrm{mg}$ de PGF $2 \alpha^{2}$ via intramuscular $(5 \mathrm{ml})$, com 12 horas de intervalo entre elas, e aplicação de uma segunda dose de $100 \mathrm{mcg}$ de gonadorelina, 48 horas depois da primeira aplicação de PGF2 $\alpha$. Durante o período de sincronização inicial, todas as vacas receberam $4 \mathrm{~kg} / \mathrm{dia}$ de concentrado.

No dia 0 (dia da segunda aplicação de GnRH), os animais foram distribuídos em dois grupos, de acordo com a quantidade de concentrado a ser fornecida diariamente, até o dia 16 do ciclo estral ( 2 vs. $8 \mathrm{~kg} / \mathrm{dia}$ de concentrado, grupos $2 \mathrm{~kg}$ e $8 \mathrm{~kg}$, respectivamente). Foi realizada uma réplica do experimento (fases 1 e 2), e, no início da segunda fase, os animais foram sincronizados novamente. Os animais que na fase 1 receberam $2 \mathrm{~kg}$ de concentrado/dia receberam $8 \mathrm{~kg}$ na fase 2 , e os que na fase 1 receberam $8 \mathrm{~kg}$ de concentrado/dia receberam $2 \mathrm{~kg}$ na fase 2 .

Foram retirados da análise os dados de duas vacas da fase 1 , que apresentaram cio antes do dia 16 , e de um animal da fase 2 , que não ovulou ao protocolo inicial de sincronização. Portanto, na fase 1 foram utilizados os dados de cinco animais, e na fase 2 os de seis. Nos do grupo $2 \mathrm{~kg}$ foram considerados os dados de quatro animais, e nos do grupo $8 \mathrm{~kg}$ foram considerados os dados de sete animais.

Os exames ultra-sonográficos foram realizados para avaliar a ovulação ao protocolo de sincronização e para acompanhar a formação do corpo lúteo e o desenvolvimento folicular.

${ }^{1}$ Cystorelin ${ }^{\circledR}$, Merial Ltd., EUA

${ }^{2}$ Lutalyse ${ }^{\circledR}$, Pfizer-Saúde Animal, Brasil 
A partir do dia 0 , foram colhidas duas amostras de sangue, diariamente, para dosagem de progesterona, uma pela manhã, antes do fornecimento do concentrado, e outra quatro horas depois. As amostras de sangue, colhidas da veia coccígea em tubos com vácuo e heparina, foram imediatamente colocadas no gelo em posição vertical. Após centrifugação a $1200 \mathrm{~g}$ por 15 minutos e separação do plasma, procedeu-se a sua armazenagem a $-20^{\circ} \mathrm{C}$, até a realização das dosagens de progesterona.

As concentrações plasmáticas de $\mathrm{P} 4$ foram determinadas com kit de radioimunoensaio em fase sólida ${ }^{3}$, validado para o uso em vacas, e as amostras processadas em dois ensaios, com coeficiente de variação intra-ensaio de 5,1 e $5,6 \%$ e CV interensaio de $5,1 \%$.

Para cada variável, testaram-se os dados quanto à normalidade dos resíduos e quanto à homogeneidade das variâncias. Se eles não atendiam às premissas para a análise, os dados foram transformados por raiz quadrada $\mathrm{e}$ reanalisados. As médias ajustadas foram reexpressas na escala original.

Os diâmetros do folículo ovulatório no final do protocolo inicial de sincronização, do corpo lúteo e do maior folículo no dia 16 do ciclo estral foram analisados pelo procedimento GLM do SAS (User's..., 1988), sendo incluídos no modelo os efeitos de tratamento e fase e a interação tratamento $\mathrm{x}$ fase.

As concentrações plasmáticas diárias de P4, imediatamente antes do fornecimento do concentrado e quatro horas depois, foram analisadas pelo procedimento Mixed do SAS (User's..., 1988), sendo incluídos no modelo os efeitos fixos de quantidade de concentrado, fase e dia de colheita e as respectivas interações, além dos efeitos aleatórios de resíduo e de vaca, dentro de cada combinação de quantidade de concentrado e fase. Admitiu-se, para as medidas repetidas, um efeito auto-regressivo de primeira ordem. A variável concentração plasmática de P4 quatro horas após o fornecimento do concentrado foi analisada depois da transformação por raiz quadrada.

\section{RESULTADOS E DISCUSSÃO}

Não se verificou diferença entre os grupos quanto ao diâmetro do folículo ovulatório ao final do protocolo de sincronização da ovulação e quanto ao diâmetro do maior folículo no dia 16 do ciclo estral (Tab. 1). Nesse dia, o diâmetro do corpo lúteo foi maior $(\mathrm{P}<0,05)$ nos animais do grupo $8 \mathrm{~kg}$.

Tabela 1. Média dos quadrados mínimos $( \pm \mathrm{EPM})$ das variáveis diâmetro do folículo ovulatório, do corpo lúteo e do maior folículo, no dia 16 do ciclo estral, nas vacas que receberam $2 \mathrm{~kg}$ ou $8 \mathrm{~kg}$ de concentrado diariamente

\begin{tabular}{lccc}
\hline \multicolumn{1}{c}{$\begin{array}{c}\text { Diâmetro } \\
(\mathrm{mm})\end{array}$} & \multicolumn{2}{c}{ Média \pm EPM } & Valor de \\
\cline { 2 - 3 } & Grupo 2kg & Grupo 8kg & P \\
\hline Diâmetro do folículo ovulatório* & $14,25 \pm 0,53 \mathrm{a}$ & $13,81 \pm 0,38 \mathrm{a}$ & 0,53 \\
& & & \\
Diâmetro do corpo lúteo no dia 16 & $20,17 \pm 0,49 \mathrm{a}$ & $21,60 \pm 0,32 \mathrm{~b}$ & 0,04 \\
Diâmetro folicular no dia 16** & $11,58 \pm 1,21 \mathrm{a}$ & $11,40 \pm 0,80 \mathrm{a}$ & 0,90 \\
\hline
\end{tabular}

*No dia 0 do experimento; **do maior folículo no dia 16 do experimento. Valores seguidos por letras distintas na mesma linha diferem entre si $(\mathrm{P}<0,05)$.

Detectou-se interação quantidade de concentrado fornecida versus dia da colheita das amostras quanto à concentração plasmática de P4 imediatamente antes do fornecimento do concentrado $(\mathrm{P}<0,05)$. Esta foi menor nos animais do grupo $8 \mathrm{~kg}$ em relação aos do grupo
$2 \mathrm{~kg}$ nos dias $10(3,84 \pm 0,48$ vs. $2,68 \pm 0,32 \mathrm{ng} / \mathrm{ml}$; $\mathrm{P}<0,05), \quad 11 \quad(4,09 \pm 0,48$ vs. $2,90 \pm 0,32 \mathrm{ng} / \mathrm{ml}$; $\mathrm{P}<0,05), \quad 14 \quad(4,92 \pm 0,48$ vs. $3,66 \pm 0,32 \mathrm{ng} / \mathrm{ml}$; $\mathrm{P}<0,05)$ e 16 do ciclo estral $(5,8 \pm 0,48$ vs. $3,74 \pm 0,32 \mathrm{ng} / \mathrm{ml} ; \mathrm{P}<0,001$; Fig. 1).

\footnotetext{
${ }^{3}$ Coat a Count ${ }^{\circledR}$ - Diagnostic Products Corporation, Los Angeles, CA
} 
Quatro horas após o fornecimento do concentrado, também foi observada interação quantidade de concentrado fornecida versus dia da colheita da amostra quanto à concentração plasmática de $\mathrm{P} 4(\mathrm{P}<0,05)$. Nas vacas do grupo $8 \mathrm{~kg}$, a concentração plasmática de progesterona foi mais baixa nos dias $9 \quad(3,98 \pm 0,49$ vs. $2.51 \pm 0,33 \mathrm{ng} / \mathrm{ml} ; \quad \mathrm{P}<0,05), \quad 10 \quad(4,59 \pm 0,49 \quad$ vs. $2,93 \pm 0,33 \mathrm{ng} / \mathrm{ml} ; \quad \mathrm{P}<0,05), \quad 11 \quad(4,44 \pm 0,49 \quad$ vs. $3,19 \pm 0,33 \mathrm{ng} / \mathrm{ml} ; \quad \mathrm{P}<0,05), \quad 14 \quad(5,37 \pm 0,49 \quad$ vs. $3,44 \pm 0,33 \mathrm{ng} / \mathrm{ml} ; \quad \mathrm{P}<0,01), \quad 15 \quad(6,05 \pm 0,49 \quad$ vs. $3,65 \pm 0,33 \mathrm{ng} / \mathrm{ml} ; \mathrm{P}<0,01)$ e 16 do ciclo estral $(5,93 \pm 0,49$ vs. $3,69 \pm 0,33 \mathrm{ng} / \mathrm{ml} ; \mathrm{P}<0,01)$ do que nas do grupo $2 \mathrm{~kg}$ (Fig. 2).

O diâmetro do folículo ovulatório ao final do protocolo de sincronização foi semelhante entre os grupos (Tab. 1), porém, nos animais do grupo $8 \mathrm{~kg}$, observou-se CL de maior diâmetro no dia 16 do ciclo estral. Green et al. (2005) verificaram correlação positiva $(r=0,66)$ entre o peso do CL e a produção de $\mathrm{P} 4$, então, o esperado seria maior concentração plasmática de $\mathrm{P} 4$ nos animais do grupo $8 \mathrm{~kg}$, pois eles tinham $\mathrm{CL}$ de maior diâmetro no dia 16. Isso não foi observado. No grupo $8 \mathrm{~kg}$, a concentração plasmática de $\mathrm{P} 4$ imediatamente antes do fornecimento do concentrado foi menor, e quatro horas depois (Fig. 1 e 2), provavelmente devido ao aumento do fluxo sangüíneo para o sistema digestivo e à conseqüente maior metabolização da progesterona, foi semelhante aos resultados de Parr et al. (1993a), observados em ovelhas, e de Vasconcelos et al. (2003), que utilizaram vacas.

Parr et al. (1993b) e Sangsritavong et al. (2002) relataram que o fluxo sangüíneo para a veia porta hepática está diretamente relacionado à IMS e que o fígado apresenta eficiência de $96 \%$ na metabolização da progesterona. Concluíram que o aumento do fluxo sangüíneo para o sistema digestivo, associado à alta eficiência do fígado em metabolizar a progesterona, explica as menores concentrações de progesterona associadas à maior ingestão de concentrado.

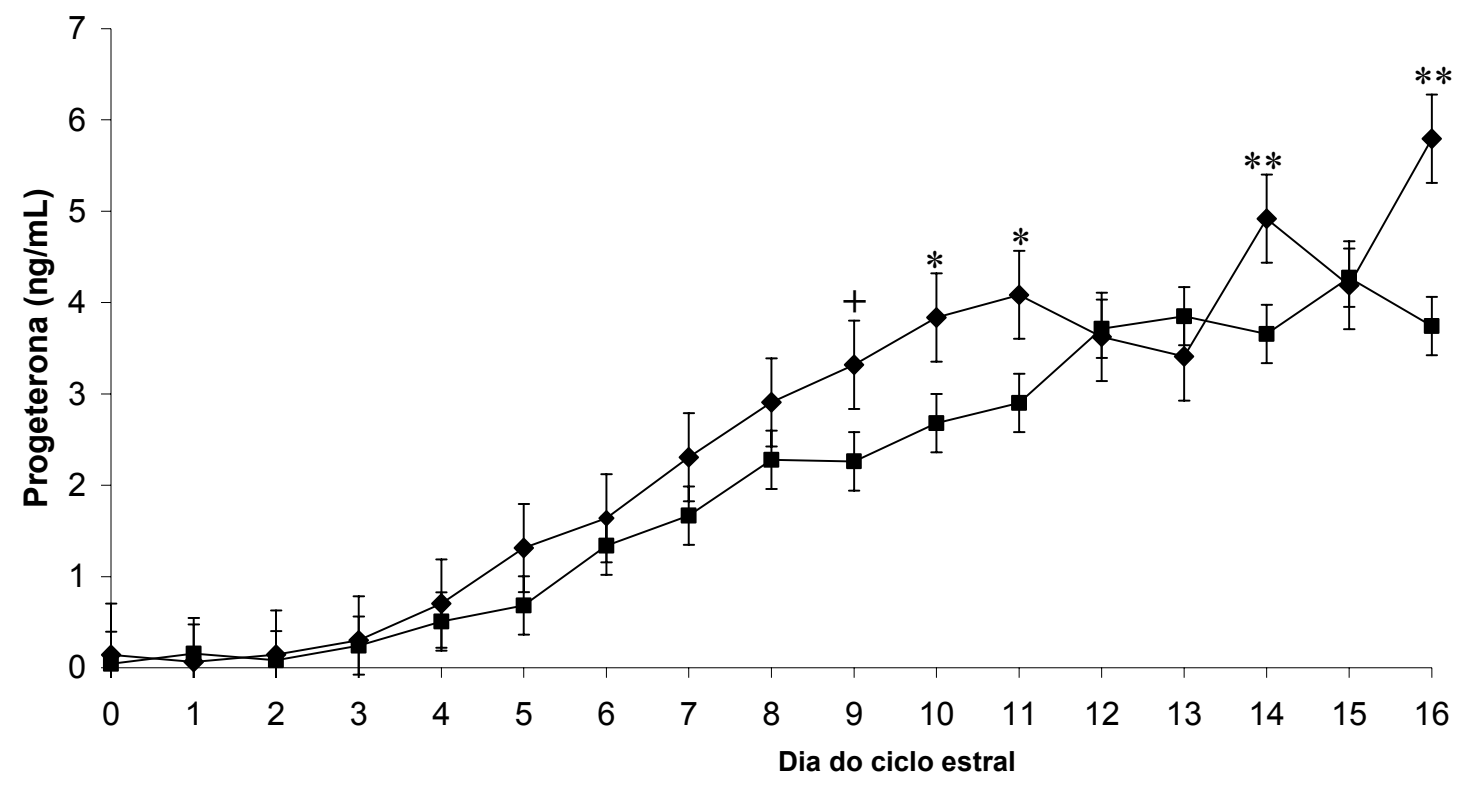

$2 \mathrm{~kg}$ de concentrado

$8 \mathrm{~kg}$ de concentrado

Figura 1. Concentração plasmática de progesterona $( \pm E P M)$ durante os primeiros 16 dias do ciclo estral, imediatamente antes da ingestão da metade do concentrado fornecido diariamente, nas vacas que receberam $2 \mathrm{~kg}$ ou $8 \mathrm{~kg}$ de concentrado.

+ Médias dos tratamentos em um mesmo tempo diferem entre si $(\mathrm{P}<0,10)$.

* Médias dos tratamentos em um mesmo tempo diferem entre si $(\mathrm{P}<0,05)$.

** Médias dos tratamentos em um mesmo tempo diferem entre si $(\mathrm{P}<0,01)$. 


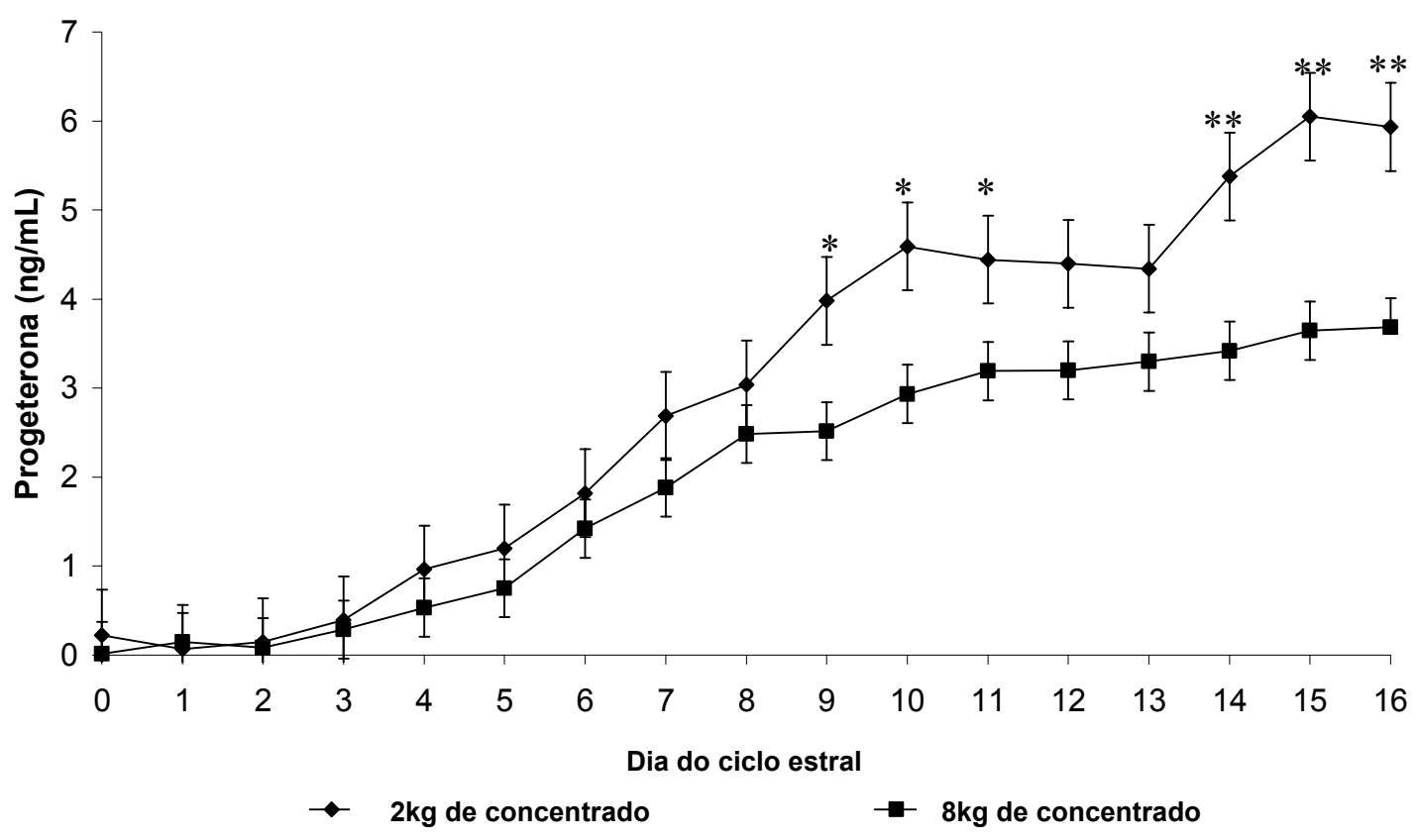

Figura 2. Concentração plasmática de progesterona ( $\pm \mathrm{EPM})$ durante os primeiros 16 dias do ciclo estral, quatro horas após a ingestão da metade do concentrado fornecido diariamente, nas vacas que receberam $2 \mathrm{~kg}$ ou $8 \mathrm{~kg}$ de concentrado.

* Médias dos tratamentos em um mesmo tempo diferem entre si $(\mathrm{P}<0,05)$.

** Médias dos tratamentos em um mesmo tempo diferem entre si $(\mathrm{P}<0,01)$.

O maior diâmetro do CL no grupo $8 \mathrm{~kg}$ pode ser explicado pela redução na concentração plasmática de progesterona e conseqüente aumento da pulsatilidade do hormônio luteinizante (Rahe et al., 1980; Bergfeld et al., 1996), responsável pelo crescimento e função do CL no início do ciclo estral, entre os dias dois e 12 (Peters et al., 1994; Niswender et al., 2000). Esse efeito estimulatório do hormônio luteinizante no desenvolvimento inicial do $\mathrm{CL}$, provavelmente foi $\mathrm{o}$ responsável pela não detecção da diferença na concentração plasmática de $\mathrm{P} 4$ entre os grupos $2 \mathrm{~kg}$ e $8 \mathrm{~kg}$, nos primeiros oito dias do ciclo estral (Fig. 1 e 2). O aumento da metabolização da P4 nos animais que receberam $8 \mathrm{~kg}$ de concentrado por dia foi, talvez, compensado pela maior produção de $\mathrm{P} 4$, atribuída ao aumento na pulsatilidade do hormônio luteinizante e conseqüente maior estimulação do $\mathrm{CL}$ na fase inicial de desenvolvimento (Peters et al., 1994; Niswender et al., 2000).

\section{CONCLUSÃO}

Os resultados confirmam os efeitos negativos da maior ingestão de concentrado na concentração plasmática de progesterona.

\section{REFERÊNCIAS BIBLIOGRÁFICAS}

BERGFELD, E.G.; KOJIMA, F.N.; CUPP, A.S. et al. Changing dose of progesterone results in sudden changes in frequency of luteinizing hormone pulses and secretion of $17 \beta$-estradiol in bovine females. Biol. Reprod., v.54, p.546553,1996 .

BUTLER, W.R. Effect of protein nutrition on ovarian and uterine physiology in dairy cattle. $J$. Dairy Sci., v.81, p.2533-2539, 1998.

DUNNE, L.D.; DISKIN, M.G.; BOLAND, M.P. et al. The effect of pre- and post-insemination 
plane of nutrition on embryo survival in beef heifers. Anim. Sci., v.69, p.411-417, 1999.

FREETLY, H.C.; FERRELL., C.L. Net uptakes of oestradiol-17B and progesterone across the portal-drained viscera and the liver of ewes. $J$. Endocrinol., v.141, p.353-358, 1994.

GREEN, M.P.; HUNTER, M.G.; MANN, G.E. Relationships between maternal hormone secretion and embryo development on day 5 of pregnancy in dairy cows. Anim. Reprod. Sci., v.88, p.179-189, 2005.

HARRISON R.O.; FORD, S.P.; YOUNG, J.W. et al. Increased milk production versus reproductive and energy status of high producing dairy cows. J. Dairy Sci., v.73, p.2749-2758, 1990.

LUCY, M. C. Reproductive loss in high producing dairy cattle: Where will it end? J. Dairy Sci., v.84, p.1277-1293, 2001.

MILLER, H.M.; FOXCROFT, G.R.; SQUIRES, J. et al. The effects of feed intake and body fatness on progesterone metabolism in ovariectomized gilts. J. Anim. Sci., v.77, p.32533261, 1999.

NISWENDER, G.D.; JUENGEL, J.L.; SILVA, P.J. et al. Mechanisms controlling the function and life span of the corpus luteum. Physiol. Rev., v.80, p.1-29, 2000.

PARR, R.A.; DAVIS, I.F.; FAIRCLOUGH, R.J. et al. Overfeeding during early pregnancy reduces peripheral progesterone concentration and pregnancy rate in sheep. J. Reprod. Fertil., v.80, p.317-320, 1987.

PARR, R.A.; DAVIS, I.F.; MILES, M.A. et al. Feed intake affects metabolic clearance rate of progesterone in sheep. Res. Vet. Sci., v.55, p.30610, 1993a.
PARR, R.A.; DAVIS, I.F.; MILES, M.A. et al. Liver blood flow and metabolic clearance rate of progesterone in sheep. Res. Vet. Sci., v.55, p.311-316, 1993b.

PETERS, K.E.; BERGFELD, E.G.; CUPP, A.S. et al. Luteinizing hormone has a role in development of fully functional corpora lutea (CL) but is not required to maintain CL function in heifers. Biol. Reprod., v.51, p.1248-1254, 1994.

RAHE, C.H.; OWENS, R.E.; FLEEGER, J.L. et al. Pattern of plasma luteinizing hormone in the cyclic cow: Dependence upon the period of the cycle. Endocrinology, v.107, p.498-503, 1980.

SANGSRITAVONG, S.; COMBS, D.K.; SARTORI, R. et al. High feed intake increases liver blood flow and metabolism of progesterone and estradiol-17ß in dairy cattle. J. Dairy Sci., v.85, p.2831-2842, 2002.

SYMONDS, H.W.; PRIME. G.R. The influence of volume of food intake by gilts on blood flow in the portal vein and clearance of progesterone from plasma. Anim. Prod., v.48, p.620-621, 1989.

USER'S guide: statistics. Version 6.02. Cary, NC: SAS Institute, 1988.

VASCONCELOS, J.L.M.; SANGSRITAVONG, S.; TSAI, S.J. et al. Acute reduction in serum progesterone concentration after feed intake in dairy cows. Theriogenology, v.60, p.795-807, 2003.

WASHBURN, S.P.; SILVIA, W.J.; BROWN, C.H. et al. Trends in reproductive performance in southeastern Holstein and Jersey DHI herds. $J$. Dairy Sci., v.85, p.244-251, 2002. 